\title{
Hypothalamic-pituitary-adrenal axis activity and early onset of cannabis use
}

\author{
Anja C. Huizink', Robert F. Ferdinand ${ }^{1,2}$, Johan Ormel ${ }^{2,3}$ \& Frank C. Verhulst' \\ Department of Child and Adolescent Psychiatry, Erasmus Medical Center, Rotterdam, the Netherlands,' Department of Psychiatry and Graduate School of \\ Behavioural and Cognitive Neurosciences, University Medical Center Groningen and University of Groningen, the Netherlands, ${ }^{2}$ and Graduate School for \\ Experimental Psychopathology, the Netherlands ${ }^{3}$
}

\begin{abstract}
Aims To identify early onset cannabis users by measuring basal hypothalamic-pituitary-adrenal (HPA) axis activity, which may be a risk factor for early onset substance use when showing low activity. Design In a prospective cohort study, adolescents who initiated cannabis use at an early age (9-12 years), those who initiated at a later age (1314 years) and those who did not use cannabis by the age of 14 were compared with respect to HPA axis activity. Setting and participants Data were used from the first and second assessment wave of the TRacking Adolescents' Individual Lives Survey (TRAILS), that included 1768 Dutch young adolescents aged 10-12 years who were followedup across a period of 2 years. Measurements Cortisol was measured in saliva samples at awakening, 30 minutes later and at 8 p.m. at age 10-12. Self-reported age at first cannabis use was used. Findings The early onset group had lower cortisol levels 30 minutes after awakening than the late onset group (OR = 0.93, 95\% CI: 0.86-0.99). Furthermore, compared to non-users, the early and late onset cannabis users had higher levels of cortisol at 8 p.m. $(\mathrm{OR}=1.25,95 \% \mathrm{CI}: 1.03-1.53$ and $\mathrm{OR}=1.21,95 \% \mathrm{CI}: 1.01-1.45$, respectively). Conclusions Some evidence was found for HPA axis hypo-activity at awakening in adolescents with early onset of cannabis use compared to late onset users, which might indicate an increased risk for early onset users of seeking stimulation to restore arousal levels by using substances.
\end{abstract}

Keywords Adolescents, cannabis, cortisol, early onset, HPA axis.

Correspondence to: Anja C. Huizink, Erasmus Medical Center Rotterdam/Sophia Children's Hospital, Department of Child and Adolescent Psychiatry, PO Box 2060, 3000 CB Rotterdam, the Netherlands. E-mail: a.c.huizink@erasmusmc.nl

Submitted 21 December 2005; initial review completed 14 February 2006; final version accepted 19 May 2006

\section{INTRODUCTION}

Adolescents who initiate cannabis use at an early age have been found to be at increased risk for later dependence [1,2], later psychosis [3], neuropsychological deficits [4] and a variety of other adverse health outcomes (e.g. [5]). In particular, adolescents who initiate cannabis use before the age of 13 may be at risk [6-8]. It is therefore important to be able to identify this high-risk group among adolescents. The search for markers that predict substance use has led many researchers to examine temperament and personality factors, such as sensationseeking (e.g. [9]). Sensation-seeking is often defined as 'the need for varied, novel and complex sensations and experiences, and the willingness to take physical and social risks for the sake of these experiences' [10]. These aspects of behaviour are associated with the activation of the hypothalamic-pituitary-adrenal (HPA) axis, which is a central component of the body's neuroendocrine response to stress. Its major end-product cortisol has profound effects on mood and behaviour. HPA axis activity may reflect a risk factor that explains part of the association between temperament, personality factors and early onset of substance use.

Several animal studies have focused on the relationship between HPA axis activity and self-administration of drugs. It has been found, for instance, that lower HPA axis activity was associated with heightened acquisition of drug self-administration in rats [11]. These findings from animal studies suggest that individuals with low responsiveness to stress may, in fact, use drugs actively to increase the arousal effects, and evoke stronger sensations that are essential for physiological comfort [12]. 
Animal studies have also shown that administration of tetrahydrocannabinol (THC), the main active compound of cannabis, enhanced the HPA axis response to a painful stimulus and increased basal concentrations of HPA axis hormones, such as adrenocorticotrophin hormone (ACTH) and corticosterone $[13,14]$. Thus, using cannabis may indeed enhance HPA axis activity.

Only few studies exist in which human HPA axis activity has been related to risk of substance use. A study of a small sample of boys whose fathers had a substance use disorder showed that the cortisol reactivity of these boys, who may be regarded as being at high risk of developing a substance use disorder, was lower when anticipating a stress task when compared to normal controls [15]. Similar results were found in a larger sample of high-risk boys, who also had a substance-abusing father [16]. To our knowledge, there have been no studies that have examined the prospective relationship between HPA axis activity and onset of cannabis use.

To study HPA axis dysregulation as a risk factor contributing to the onset of substance use, longitudinal data are needed covering a period in which adolescents start to experiment with substance use. Salivary cortisol is now used commonly as an index of HPA axis activity, because it is non-invasive and comparatively inexpensive to collect and analyse [17]. Cortisol concentrations follow a circadian rhythm [18]. Cortisol levels start to rise after awakening, peak at about half an hour after awakening $[19,20]$ and decrease during the day.

Other aspects that should be taken into account when studying cortisol levels are gender and pubertal development. There is some evidence for gender differences in basal levels of cortisol. Klimes-Dougan et al . [21] reported higher salivary cortisol levels in female than in male controls at mid-day and late afternoon. Gonadal steroids are known to interact with the HPA axis, and oestrogens in particular have been shown to stimulate HPA axis activity [22-24]. Thus, when studying the relationship between HPA axis activity and substance use onset, pubertal stage should also be taken into account.

The present study examined whether adolescents who initiated cannabis use before the age of 13 (9-12 years), those who initiated cannabis use at a later age (1314 years) and those who did not use any substance by the age of 14 differed with respect to HPA axis activity. We hypothesized that hypo-activity of the HPA axis is related to an increased risk of early onset cannabis use.

\section{METHODS}

\section{Participants}

The TRacking Adolescents' Individual Lives Survey (TRAILS) is a prospective cohort study of Dutch early adolescents aged 10-12 years, who were followed-up across a period of 2 years and who will have several follow-up measurements in the future. Data collection at the first and second assessment waves took place from March 2001 to July 2002, and from September 2003 to May 2004, respectively. The TRAILS target sample comprised young adolescents from five municipalities in the north of the Netherlands, including both urban and rural areas [25]. The average age at the first assessment was 11.1 years and at the second assessment was 13.6 years.

The sample selection involved two steps. First, the municipalities were requested to provide names and addresses of all inhabitants born between 10 January 1989 and 30 September 1990 (first two municipalities) or between 10 January 1990 and 30 September 1991 (last three municipalities), which yielded 3483 names. Simultaneously, primary schools (including schools for special education) within these municipalities were approached with a request to participate. School participation was a prerequisite for eligible adolescents and their parents to be approached by TRAILS, with the exception of adolescents who already attended secondary schools $(<1 \%)$, who were contacted without involving their schools. Of the 135 primary schools within the municipalities, 122 (90.4\%) schools agreed to participate, accommodating $90.3 \%$ of the adolescents. Secondly, if schools agreed to participate, parents (or guardians) received two brochures, one for themselves and one for their adolescents, with information about the study. In addition, a TRAILS staff member visited the schools to inform eligible adolescents about the study. More details about the procedure have been published elsewhere [25].

The exclusion criteria were: (1) adolescent incapable of participating because of intellectual disability or a serious physical illness or handicap; (2) Dutch-speaking parent or parent surrogate not available, and not feasible to administer a part of the measurements in parent's own language. Of all subjects who were approached ( $n=3145), 6.7 \%$ were excluded. Of the remaining 2935 young adolescents, $76.0 \%$ were included in the study $(n=2230$, mean age 11.09 years, SD $0.55,50.8 \%$ female). A high response rate $(96 \%)$ was yielded at the second assessment. Saliva samples were received at the first assessment wave of 1768 children $(79.3 \%$ of all TRAILS participants).

\section{Procedure}

At the first assessment, well-trained interviewers visited one of the parents or guardians in their homes to administer an interview covering a wide range of topics. In addition, the parent was asked to complete a questionnaire. Children were measured at school, where they completed questionnaires in groups under the supervision of one or 
more TRAILS assistants. In addition, information-processing capabilities (neurocognitive tasks), intelligence and a number of biological parameters (including weight and height) were assessed individually at school. Saliva samples were collected at home using the Salivette sampling device (Sarstedt, Nümbrecht, Germany), which was given to the parent at the parent interview, accompanied by verbal and written instructions. Teachers completed a brief questionnaire for each TRAILS child in their class.

The second assessment involved only self-report questionnaires, to be completed by the children (or young adolescents), their parents and their teachers. The adolescents completed their questionnaires at school, supervised by TRAILS assistants.

\section{Measures}

Cannabis use

Cannabis use by the participant was measured at the first and second assessments by four self-report items. The first question concerned the age of first cannabis use, with the following answer categories: never used, 9 years or younger, 10, 11, 12, 13 or 14 years). Children were also asked about life-time use, use in the last year and use in the last month with the following questions: 'How often have you used cannabis in your life/in the last year/in the last months', with answer categories: 'I have never used', 'used it once', 'used it twice', 'three times', ..., '10 times', '11-19 times', '20-39' times, ‘40 times or more’). For the present study we used the age at first use and formed three groups: (1) early onset, i.e. age at first use was 9-12 years, (2) later onset, i.e. age at first use was 13-14 years and (3) non-users, i.e. adolescents who had not experimented with cannabis use by the age of 14 at the second assessment.

\section{Cortisol}

Collection of salivary cortisol is a relatively stress-free approach that avoids confounding by stress responses, e.g. as induced by venipuncture, and correlations between saliva cortisol levels and serum cortisol concentrations are high [26]. Cortisol concentrations in the collected samples depend strongly on the time of the day that samples are taken. For the present study we focused on cortisol measures taken by means of Salivettes shortly after awakening, still lying in bed (cortisol 1), 30 minutes after awakening (cortisol 2) and at 8 p.m. (cortisol 3) to cover various time-points during the circadian rhythm that have been found to be relevant [27]. TRAILS participants collected their saliva at home, using the Salivette sampling device (Sarstedt, Rommelsdorfer Str, D-51588 Nümbrecht, Germany), which was given to the parent at the parent interview, accompanied by verbal and written instructions. The Salivette tube consists of a plastic sampling vessel with a suspended insert containing a sterile neutral cotton wool swab that has to be chewed for about 45 seconds and then returned to the insert. A recent study by Shirtcliff et al . [41] showed that results for salivary cortisol were not affected by the use of Salivettes. Both the sampling and the preceding day should be normal (school) days, without special events or stressful circumstances. Because the schools participating in TRAILS started at approximately the same time, the samplingtime variation of the morning samples among the children is limited and the estimated corresponding times are 07.00 hours for the first sample (group 1) and 07.30 hours for the second sample (group 2). Children were instructed not to collect saliva when they were ill, had a cold, had a headache or were menstruating. Furthermore, they were requested not to take any medication, if possible. Any deviations from this protocol, either in terms of sampling times or in terms of other requirements, were indicated on an accompanying form. Concerning the sampling procedure itself, subjects were instructed to keep a glass of water next to their bed and to rinse their mouth thoroughly with tap water before sampling saliva, and not to consume sour products or brush their teeth shortly beforehand. Saliva samples were stored by the participants in their freezer directly after sampling and mailed to the institute as soon as possible (but not on Fridays and Saturdays, in order to prevent unnecessary delay due to the weekend). The saliva samples were stored at $-20^{\circ} \mathrm{C}$ until analysis. Previous studies suggest that salivary cortisol levels are stable for prolonged periods of time at $-20^{\circ} \mathrm{C}$ [28]. Participants who did not return the Salivettes within 2 months were sent a reminder letter [29].

A time-resolved fluorescence immunoassay was used in order to determine the cortisol concentration in the saliva samples. After thawing, saliva samples were centrifuged at $2000 \boldsymbol{g}$ for 10 minutes, which resulted in a clear supernatant of low viscosity; $100 \mathrm{ml}$ of saliva were used for duplicate analysis. Cortisol levels were determined employing a competitive solid-phase time-resolved fluorescence immunoassay with fluorometric end-point detection (DELFIA = dissociation-enhanced lanthanide fluorescent immunoassays). Ninety-six-well Maxisorb microtitre plates (Nunc, Wiesbaden, Germany) were coated with rabbit-anti-ovine immunoglobulin. After an incubation period of 48 hours at $4^{\circ} \mathrm{C}$ plates were washed three times with washbuffer $(\mathrm{pH}=7.4$, containing sodium phosphate and Tween-40). In the next step the plates were coated with an ovine anti-cortisol antibody and incubated for 48 hours at $4^{\circ} \mathrm{C}$. Synthetic saliva mixed with cortisol in a range from 0 to $100 \mathrm{nmol} / \mathrm{l}$ served as standards. Standards, controls (saliva pools) and samples were given in duplicate wells. Fifty microlitres of biotin-conjugated cortisol was added and after 
30 minutes of incubation the non-binding cortisol/ biotin-conjugated cortisol was removed by washing (three times). Two hundred microlitres of europiumstreptavidin (Wallac Oy, Turku, Finland) was added to each well and after 30 minutes and six washings $200 \mu \mathrm{l}$ enhancement solution was added (Pharmacia, Freiburg, Germany). Within 15 minutes on a shaker the enhancement solution induced fluorescence, which can be detected with a DELFIA fluorometer (Wallac). A standard curve was generated and the cortisol concentration of the samples was calculated with a computer-controlled program. The intra-assay coefficient of variation was between 4.0 and $6.7 \%$, and the corresponding interassay coefficients of variation were between 7.1 and 9.0\% [29].

Cortisol was assessed in 1768 children. We excluded 22 children because of the use of corticosteroid-containing medication. We next analysed the effect of other remarks concerning the sampling procedure that parents indicated on the accompanying form (such as stressful events during the day or deviations in terms of sampling times), and found no further reasons for exclusion. For each time-point, cortisol values that were above 3 standard deviations (SD) of the mean were excluded from the analysis in order to reduce the impact of outliers (cortisol 1, 21 excluded; cortisol 2, 11 excluded; cortisol 3, 18 excluded). After this exclusion, cortisol levels followed a normal distribution.

Pubertal stage

Pubertal stage was assessed using schematic drawings of secondary sex characteristics associated with the five Tanner stages of pubertal development [30], a method that is used widely and has demonstrated good reliability and validity [31,32]. Stage of pubertal development was assessed in the parent interview using schematic drawings of secondary sex characteristics associated with the five standard Tanner stages of pubertal development [33]. Tanner stages are a widely accepted standard for assessment of physical development, and have demonstrated good reliability, validity and parent-child agreement [32,33]. A parent (usually the mother) was provided with gender-appropriate sketches, and asked to select which of the sketches 'looked most like the child'. Based on parent ratings, children were classified into five stages of puberty, in which stage 1 corresponds to infantile and stage 5 to complete puberty [34].

\section{Statistical analyses}

We checked whether non-responders differed from responders on various background characteristics by means of $\chi^{2}$ and $t$-tests. Descriptives for prevalence of cannabis use and age at onset and for the different cortisol measures (cortisol 1, cortisol 2 and cortisol 3) were computed. Next, three multivariate logistic regression analy- ses were conducted with age of onset of cannabis use (early onset versus no use; later onset versus no use; early versus later onset) as dependent variable, and cortisol 1, cortisol 2 and cortisol 3 as multivariate predictors in each model. All analyses were adjusted for gender and Tanner stage by including them as covariates in the regression models. Odds ratios (ORs) and their 95\% confidence intervals (CI) were estimated. The analyses were carried out using SPSS version 12.0, and $P<0.05$ (two-tailed) was used to determine statistical significance.

\section{RESULTS}

\section{Non-responders analysis}

Responders and non-responders did not differ with respect to the proportion of single-parent families or the prevalence of teacher-rated problem behaviour. Furthermore, no differences between responders and nonresponders were found regarding associations between socio-demographic variables and mental health outcomes [25]. Non-responders did not differ from responders in terms of gender [in the non-responders group $48.48 \%$ were male compared to $49.43 \%$ male in the responders group, $\chi^{2}(\mathrm{df}=1)=0.13 ; P=0.72$ ] or pubertal development (average Tanner score $=1.92$ versus 1.86, $t=-1.39 ; P=0.16)$, but non-responders were slightly older (11.16 years versus 11.08 years, $t=$ -3.08; $P=0.002)$.

\section{Descriptives}

Descriptive information regarding the prevalence of cannabis use and the age at onset of cannabis use for cortisol 1 , cortisol 2 and cortisol 3 is presented in Table 1 for all participants $(n=1441)$ who had complete data on all variables included in the analyses. The cortisol samples were only modestly intercorrelated ( $r$ s ranging from 0.17 to 0.26 , Ps $<0.005$ ), which indicates that multi-collinearity is most probably not at stake. Background characteristics of the three analytical groups are presented in Table 2.

\section{Logistic regression analyses}

Results of the logistic regression analyses are presented in Table 3. For one early starter and two late starters we had missing data on pubertal stage, and therefore these participants were excluded from the logistic regression analyses. The results show that both early and later onset of cannabis use were associated with an increased risk of higher cortisol levels at 8 p.m. when compared to nonusers $(\mathrm{OR}=1.25,95 \% \mathrm{CI}=1.03-1.53$ and $\mathrm{OR}=1.21$, $95 \% \mathrm{CI}=1.01-1.45$, respectively). When early and later starters of cannabis use were compared, the early onset group had statistically significant lower cortisol levels 
Table 1 Descriptives of cortisol and prevalence and age of onset of cannabis use.

\begin{tabular}{llll}
\hline & Mean & SD & Range \\
\hline Cortisol $1 \mathrm{nmol} / \mathrm{l}$ & $11.53(n=1441)$ & 4.67 & $0.71-29.42$ \\
Cortisol $2 \mathrm{nmol} / \mathrm{l}$ & $15.26(n=1441)$ & 6.40 & $0.22-38.42$ \\
Cortisol $3 \mathrm{nmol} / \mathrm{l}$ & $1.94(n=1441)$ & 1.32 & $0.01-8.17$ \\
& $\%$ at first assessment & $\%$ at second assessment & Age of onset \\
Cannabis use & $1.2 \%(n=27)$ & $7.2 \%(n=148)$ & $9-12$ years: $n=45$ \\
& & & $13-14$ years: $n=61$ \\
\hline
\end{tabular}

Table 2 Background characteristics of the three groups: early starters (9-12 years old), late starters (13-14 years old), nonusers.

\begin{tabular}{|c|c|c|c|}
\hline & Earlystarters & Latestarters & Non-users \\
\hline Male gender & $66.7 \%$ & $53.8 \%$ & $48.3 \%$ \\
\hline \multicolumn{4}{|l|}{$\begin{array}{l}\text { DSM-orientated } \\
\text { scale score }\end{array}$} \\
\hline \multicolumn{4}{|l|}{$\begin{array}{l}\text { Oppositional } \\
\text { defiant disorder }\end{array}$} \\
\hline Mean (SD) & $2.9(2.5)$ & $3.3(2.3)$ & $2.0(1.9)$ \\
\hline \multicolumn{4}{|l|}{$\begin{array}{l}\text { DSM-orientated } \\
\text { scale score }\end{array}$} \\
\hline \multicolumn{4}{|l|}{ Conduct disorder } \\
\hline Mean (SD) & $3.8(4.7)$ & $3.4(3.2)$ & $1.5(2.2)$ \\
\hline
\end{tabular}

30 minutes after awakening $(\mathrm{OR}=0.93,95 \% \mathrm{CI}=0.86-$ $0.99)$.

\section{DISCUSSION}

Various studies have shown that substance use before the age of 13 years predicts substance abuse problems at later ages [6-8]. It is therefore of importance to obtain a better understanding of the processes that act to promote early use. The present study was the first that examined prospectively whether HPA axis basal activity predicts early onset cannabis use. Interestingly, early onset and later onset of cannabis use could be differentiated by means of cortisol levels 30 minutes after awakening. Early onset of cannabis use was related to lower levels of cortisol at this time of day, when compared to adolescents with later onset. Thus, adolescents with early onset of cannabis use had a slightly blunted cortisol awakening response that was different from non-users and, in particular, from adolescents with a later onset of cannabis use. Furthermore, our results showed a trend towards a significant association between low cortisol levels 30 minutes after awakening and early onset cannabis use compared to non-users. However, statistically significant associations were found between higher, instead of lower, cortisol levels at 8 p.m. and either early onset or later onset of can- nabis use, compared to non-users. In the evening, the expected decrease in cortisol levels due to its circadian rhythm was most profound for non-users, whereas both early and later onset of cannabis use were related to higher cortisol levels.

The awakening response of cortisol is regarded as a marker of basal cortisol physiology, and has recently received considerable attention (see [27] for a review). This cortisol awakening response has been shown to be an intraindividually stable phenomenon. For instance, Clow et al . [27] found an intracorrelation of $r=0.71$ for the awakening response over 2 consecutive days. Moreover, this awakening response seems able to reveal subtle interindividual changes in HPA regulation [35], appearing to be under a different regulatory influence than the remaining diurnal profile. For example, the cortisol awakening response is under genetic influences, which explain $40-59 \%$ of the variance $[20,36]$, whereas evening levels of cortisol are mainly to due environmental influences $[20,36]$. Thus, adolescents with early onset of cannabis use may have a different genetic constellation than adolescents with a later onset of cannabis use, which may relate to their blunted cortisol awakening response. In line with the stimulation-seeking theory [10], their lower arousal in the morning may put them at risk of seeking stimulation, perhaps by using substances in order to restore their arousal levels to an optimal or normal level.

Various studies have shown that temperament and personality factors, such as sensation-seeking (e.g. $[9,37])$, are related to early onset of cannabis use. The relation between cortisol and sensation-seeking behaviour has also been tested and most studies report an inverse association, with lower cortisol levels for (mostly male) individuals with high sensation-seeking levels [3840]. Thus, hypo-activity of the HPA axis under basal conditions may be a risk factor that explains part of the relationship between some aspects of temperament and personality and early onset of substance use. Hypoactivity of the HPA axis has also been reported for boys with higher levels of externalizing problem behaviours in normal, at-risk and psychiatric groups [41]. 
Table 3 Comparisons between early starters, late starters and non-users with regard to cortisol measures at awakening (cortisol 1), 30 minutes after awakening (cortisol 2) and at 8 p.m. (cortisol 3).

\begin{tabular}{|c|c|c|c|c|}
\hline \multicolumn{5}{|l|}{ Cortisol values } \\
\hline Early starters versus non-users & $\begin{array}{l}\text { Early starters } \\
(n=44) \\
\text { mean }(S D)\end{array}$ & $\begin{array}{l}\text { Non-users } \\
(n=1338) \\
\text { mean }(S D)\end{array}$ & OR & $(95 \% \mathrm{CI})$ \\
\hline Cortisol 1 & $11.11(4.78)$ & $11.54(4.72)$ & 1.01 & $0.95-1.08$ \\
\hline Cortisol 2 & $13.50(6.28)$ & $15.40(6.54)$ & 0.95 & $0.91-1.00 \dagger$ \\
\hline Cortisol 3 & $2.23(1.56)$ & $1.92(1.30)$ & 1.25 & $1.03-1.53^{*}$ \\
\hline Late starters versus non-users & $\begin{array}{l}\text { Late starters } \\
(n=59) \\
\text { mean }(S D)\end{array}$ & $\begin{array}{l}\text { Non-users } \\
(n=1338) \\
\text { mean }(S D)\end{array}$ & OR & $(95 \% \mathrm{CI})$ \\
\hline Cortisol 1 & $11.76(5.16)$ & $11.54(4.72)$ & 0.99 & $0.93-1.05$ \\
\hline Cortisol 2 & $16.06(5.78)$ & $15.40(6.54)$ & 1.02 & $0.97-1.06$ \\
\hline Cortisol 3 & $2.31(1.60)$ & $1.92(1.30)$ & 1.21 & $1.01-1.45^{*}$ \\
\hline Early starters versus late starters & $\begin{array}{l}\text { Early starters } \\
(n=44) \\
\text { mean }(S D)\end{array}$ & $\begin{array}{l}\text { Late starters } \\
(n=59) \\
\text { mean }(S D)\end{array}$ & OR & $(95 \%$ CI) \\
\hline Cortisol 1 & $11.11(4.78)$ & $11.76(5.16)$ & 1.04 & $0.95-1.15$ \\
\hline Cortisol 2 & $13.50(6.28)$ & $16.06(5.78)$ & 0.93 & $0.86-0.99^{*}$ \\
\hline Cortisol 3 & $2.23(1.56)$ & $2.31(1.60)$ & 1.03 & $0.79-1.33$ \\
\hline
\end{tabular}

${ }^{*} P<0.05 ; \dagger P<0.10$; OR: odds ratio; CI: confidence interval; analyses are adjusted for gender and Tanner stage of pubertal development.

Several authors have speculated on the significance of hypo-arousal of the HPA axis, which may either reflect an under-aroused, over-regulated HPA axis or an increased threshold for stress [42-44]. The under-aroused, overregulated HPA axis might be due to an increased negative feedback inhibition which could be caused by continuous exposure to adverse events, such as maltreatment or post-traumatic stress disorders [45]. Of interest for the present study is the 'increased threshold for stress' theory, which would imply that these individuals would seek stimulation to induce a physiological response, i.e. to arouse their HPA axis activity, in line with the sensationseeking theory. Stimulation may be derived from either substance use or, for instance, externalizing behaviours, which explains the similar findings with regard to HPA axis activity across early onset substance users and adolescents with externalizing behaviour problems.

Another finding of the present study is the blunted decrease during the evening for adolescents with early and later onset of cannabis use, which may be explained by environmental factors common to both groups of users, because variations in evening levels of cortisol were found to be due mainly to environmental influences $[20,36]$. These influences may include adverse interaction with peers, parent-child conflict and school problems during the day, because these factors have all been found to predict substance use [46-48]. Most of the early and late onset users in our study have used cannabis only a few times in their lives $(53 \%$ of early onset users and $65 \%$ of late onset users have used cannabis one to three times) and these users show even lower prevalence rates of actual use in the last 4 weeks (of the early and late onset groups, $76 \%$ and $82 \%$ did not use or used cannabis only once, respectively). Therefore, we do not expect that the elevated cortisol levels at 8 p.m. were caused by current use of cannabis in our sample.

The present study is not without limitations. First, a general population sample is representative but is characterized by low prevalence rates of cannabis use, especially because of our young age groups, which may have influenced the results. However, because of the importance of studying correlates and predictors of cannabis use at an early age, our results contribute to the identification of adolescents at risk. Secondly, the cortisol response to stress was not assessed, while stress reactivity may be a key factor in the link between HPA axis activity and early onset of cannabis use.

In conclusion, the present study shows some evidence for hypo-activity of the HPA axis in adolescents with early onset of cannabis use, and a blunted decrease in cortisol levels in the evening for both early and later onset users. Further research is warranted to differentiate between early onset and later onset users on a variety of biological and environmental risk factors, because early onset users in particular form a high-risk group that needs special attention.

\section{Acknowledgement}

This research is part of the TRacking Adolescents' Individual Lives Survey (TRAILS). Participating centres of 
TRAILS include various Departments of the University of Groningen, the Erasmus Medical Center of Rotterdam, the University of Nijmegen, University of Leiden and the Trimbos Institute, the Netherlands. TRAILS is supported financially by grants from the Netherlands Organization for Scientific Research (GB-MW 940-38-011, GB-MAGW 480-01-006, GB-MAGW 457-03-018, GB-MAGW 175.010.2003.005, ZonMw 100-001-001 'Geestkracht' Program, ZonMw 60-60600-98-018), the Sophia Foundation for Medical Research (project 301 and 393), the Ministry of Justice and by the participating centres. The authors would like to thank Frouke Sondeijker and Kirstin Greaves-Lord for their contribution to the data-collection of the present study.

\section{References}

1. Kandel D. B. Epidemiological and psychosocial perspectives on adolescent drug use. J Am Acad Child Psychiatry 1982; 21: 328-47.

2. Stein J. A., Newcomb M. D., Bentler P. M. An 8-year study of multiple influences on drug use and drug use consequences. J Pers Soc Psychol 1987; 53: 1094-105.

3. Semple D. M., McIntosh A. M., Lawrie S. M. Cannabis as a risk factor for psychosis: systematic review. J Psychopharmacol 2005; 19: 187-94.

4. Pope H. G. Jr, Gruber A. J., Hudson J. I., Cohane G., Huestis M. A., Yurgelun-Todd D. Early onset cannabis use and cognitive deficits: what is the nature of the association? Drug Alcohol Depend 2003; 69: 303-10.

5. Kalant H. Adverse effects of cannabis on health: an update of theliteraturesince 1996.ProgNeuropsychopharmacolBiol Psychiatry 2004; 28: 849-63.

6. Hawkins J. D., Catalano R. F., Miller J. Y. Risk and protective factors for alcohol and other drug problems in adolescence and early adulthood: implications for substance abuse prevention. Psychol Bull 1992; 112: 64-105.

7. Kandel D. B., Davies M., Karus D., Yamaguchi K. The consequences in young adulthood of adolescent drug involvement. An overview. Arch Gen Psychiatry 1986; 43: 746-54.

8. Robins L., Przybeck T. R. Age of onset of druguse as a factor in drug and other disorders . . IIDA Research Monograph no. 54. Washington, DC: US Government Printing Office; 1985.

9. Martin C. A., Kelly T. H., Rayens M. K., Brogli B. R., Brenzel A., Smith W. J. et al. Sensation seeking, puberty, and nicotine, alcohol, and marijuana use in adolescence. J Am Acad Child Adolesc Psychiatry 2002; 41: 1495-502.

10. Zuckerman M., Neeb M. Sensation seeking and psychopathology. Psychiatry Res 1979; 1: 255-64.

11. Kosten T. A., Ambrossio E. HPA axis function and drug addictive behaviors. insight from studies with Lewis and Fischer 344 inbred rats. Psychoneuroendocrinology 2002; 27: 35-69.

12. Majewska M. D. HPA axis and stimulant dependence: an enigmaticrelationship.Psychoneuroendocrinology 2002;27: $5-12$.

13. Finn D. P., Beckett S. R., Roe C. H., Madjd A., Fone K. C., Kendall D. A. et al. Effects of coadministration of cannabinoids and morphine on nociceptive behaviour, brain monoamines and HPA axis activity in a rat model of persistent pain. Eur J Neurosci 2004; 19: 678-86.
14. Manzanares J., Corchero J., Fuentes J. A. Opioid and cannabinoid receptor-mediated regulation of the increase in adrenocorticotropin hormone and corticosterone plasma concentrations induced by central administration of delta (9)-tetrahydrocannabinol in rats. Brain Res 1999; 39: 1739.

15. Moss H. B., Vanyukov M. M., Martin C. S. Salivary cortisol responses and the risk for substance abuse in prepubertal boys. Biol Psychiatry 1995; 38: 547-55.

16. Dawes M. A., Dorn L. D., Moss H. B., Yao J. K., Kirisci L., Ammerman R. T. et al. Hormonal and behavioural homeostasis in boys at risk for substance abuse. Drug Alcohol Depend 1999; 55: 165-76.

17. Schwartz E. B., Granger D. A., Susman E. J., Gunnar M. R., Laird B. Assessing salivary cortisol in studies of child development. Child Dev 1998; 69: 1503-13.

18. Weitzman E. D., Fukushima D., Nogeire C., Roffwarg H., Gallagher T. F., Hellman L. Twenty-four hour pattern of the episodic secretion of cortisol in normal subjects. J Clin Endocrinol Metab 1971; 33: 14-22.

19. Pruessner J. C., Wolf O. T., Hellhammer D. H., BuskeKirschbaum A., von Auer K., Jobst S. et al. Free cortisol levels after awakening: a reliable biological marker for the assessment of adrenocortical activity. Life Sci 1997; 61: 2539-49.

20. Wüst S., Federenko I., Hellhammer D. H., Kirschbaum C. Genetic factors, perceived chronic stress, and the free cortisolresponse to awakening. Psychoneuroendocrinology 2000; 25: $707-20$.

21. Klimes-Dougan B., Hastings P. D., Granger D. A., Usher B. A., Zahn-Waxler C. Adrenocortical activity in at-risk and normally developing adolescents: individual differences in salivary cortisol basal levels, diurnal variation, and responses to social challenges. Dev Psychopathol 2001; 13: 695-719.

22. Burgess L. H., Handa R. J. Chronic estrogen-induced alterations in adrenocorticotropin and corticosterone secretion, and glucocorticoid receptor-mediated functions in female rats. Endocrinology 1992; 131: 1261-9.

23. Handa R. J., Burgess L. H., Kerr J. E., O’Keefe J. A. Gonadal steroid hormone receptors and sex differences in the hypothalamo-pituitary-adrenal axis. Horm Behav 1994; 28 : 464-76.

24. Keiss W., Meidert A., Dressendorfer K., Schriever K., Kessler U., Konig A. et al. Salivary cortisol levels throughout childhood and adolescence: relation with age, pubertal stage, and weight. Pediatr Res 1995; 37: 502-6.

25. de Winter A. F., Oldehinkel A. J., Veenstra R., Brunnekreef J. A., Verhulst F. C., Ormel J. Evaluation of non-response bias in mental health determinants and outcomes in a large sample of pre-adolescents. Eur J Epidemiol 2005; 20 : $173-$ 81.

26. Kirschbaum C., Hellhammer D. H. Salivary cortisol in psychoneuroendocrine research. recent developments and applications. Psychoneuroendocrinology 1994; 19: 313-33.

27. Clow A., Thorn L., Evans P., Hucklebridge F. The awakening cortisol response: methodological issues and significance. Stress 2004; 7: 29-37.

28. Aardal E., Holm A. C. Cortisol in saliva-reference ranges and relation to cortisol in serum. Eur J Clin Chem Clin Biochem 1995; 33: 927-32.

29. Rosmalen J. G., Oldehinkel A. J., Ormel J., de Winter A. F., Buitelaar J. K., Verhulst F. C. Determinants of salivary cortisol levels in 10-12 year old children; a population-based 
study of individual differences. Psychoneuroendocrinology 2005; 30: 483-95.

30. Tanner J. Growthat adolescence. With ageneralconsideration of theeffectsof heredityandenvironmentalfactorsupongrowth andmaturation from birth tomaturity .Oxford: Blackwell Scientific Publications; 1962.

31. Brooks-Gunn J., Warren M. P., Rosso J., Gargiulo J. Validity of self-report measures of girls' pubertal status. Child Dev 1987; 58: 829-41.

32. Dorn L., Susman E., Nottelman E., Inoff-Germain E., Chrousos G. Perceptions of puberty: adolescent, parent, and health care personnel. Dev Psychopathol 1990; 26: 322-9.

33. Marshall W. A., Tanner J. M. Variations in pattern of pubertal changes in girls. Arch Dis Child 1969; 44: 291-303.

34. Tanner J. M., Whitehouse R. H. Atlas of children's growth: normal variation and growth disorders . London: Academic Press: 1982.

35. Schmidt-Reinwald A., Pruessner J. C., Hellhammer D. H., Federenko I., Rohleder N., Schurmeyer T. H. et al. The cortisol response to awakening in relation to different challenge tests and a 12-hour cortisol rhythm. Life Sci 1999; 64: 1653-60.

36. Bartels M., Van den Berg M., Sluyter F., Boomsma D. I., de Geus E. J. Heritability of cortisol levels: review and simultaneous analysis of twin studies. Psychoneuroendocrinology 2003; 28: 121-37.

37. Wills T. A., Dishion T. J. Temperament and adolescent substance use: a transactional analysis of emerging selfcontrol. J Clin Child Adolesc Psychol 2004; 33: 69-81.

38. Wang S., Mason J., Charney D., Yehuda R., Riney S., Southwick S. Relationships between hormonal profile and novelty seeking in combat-related posttraumatic stress disorder. Biol Psychiatry 1997; 41: 145-51.

39. Rosenblitt J. C., Soler H., Johnson S. E., Quadagno D. M. Sensation seeking and hormones in men and women: exploring the link. Horm Behav 2001; 40: 396-402.
40. King R. J., Jones J., Scheuer J. W., Curtis D., Zarcone V. P. Plasma cortisol correlates of impulsivity and substance abuse. Person Individ Diff 1990; 11: 287-91.

41. Shirtcliff E. A., Granger D. A., Booth A., Johnson D. Low salivary cortisol levels and externalizing behavior problems in youth. Dev Psychopathol 2005; 17: 167-84.

42. Gunnar M. R., Vazquez D. M. Low cortisol and a flattening of expected daytime rhythm: potential indices of risk in human development. Dev Psychopathol 2001; 13: 515-38.

43. Heim C., Ehlert U., Hellhammer D. H. The potential role of hypocortisolism in the pathophysiology of stress-related bodily disorders. Psychoneuroendocrinology 2000; 25: 135.

44. Kruesi M. J., Schmidt M. E., Donnelly M., Hibbs E. D., Hamburger S. D. Urinary free cortisol output and disruptive behavior in children. J Am Acad Child Adolesc Psychiatry 1989; 28: 441-3

45. Shea A., Walsh C., Macmillan H., Steiner M. Child maltreatment and HPA axis dysregulation: relationship to major depressive disorder and post traumatic stress disorder in females. Psychoneuroendocrinology 2005; 30: 162 78

46. Von Sydow K., Lieb R., Pfister H., Hofler M., Wittchen H. U. Use, abuse and dependence of ecstasy and related drugs in adolescents and young adults-a transient phenomenon? Results from a longitudinal community study. Drug Alcohol Depend 2002; 66: 147-59.

47. Wills T. A., Resko J. A., Ainette M. G., Mendoza D. Role of parent support and peer support in adolescent substance use: a test of mediated effects. Psychol Addict Behav 2004; 18: 122-34.

48. Van den Bree M. B., Pickworth W. B. Risk factors predicting changes in marijuana involvement in teenagers. Arch Gen Psychiatry 2005; 62: 311-9. 